\title{
Newly observed $\Omega(2012)$ state and strong decays of the low-lying $\Omega$ excitations
}

\author{
Zuo-Yun Wang, ${ }^{1}$ Long-Cheng Gui, ${ }^{1,2,3, *}$ Qi-Fang Lü, ${ }^{1,2,3, \dagger}$ Li-Ye Xiao, ${ }^{4,5}$ and Xian-Hui Zhong ${ }^{1,2,3, \ddagger}$ \\ ${ }^{1}$ Department of Physics, Hunan Normal University, Changsha 410081, China \\ ${ }^{2}$ Key Laboratory of Low-Dimensional Quantum Structures and Quantum Control of Ministry of Education, \\ Changsha 410081, China \\ ${ }^{3}$ Synergetic Innovation Center for Quantum Effects and Applications(SICQEA), Hunan Normal University, \\ Changsha 410081, China \\ ${ }^{4}$ Center of High Energy Physics, Peking University, Beijing 100871, China \\ ${ }^{5}$ School of Physics and State Key Laboratory of Nuclear Physics and Technology, \\ Peking University, Beijing 100871, China
}

(Received 31 October 2018; published 20 December 2018)

\begin{abstract}
Stimulated by the newly discovered $\Omega(2012)$ resonance at Belle II, in this work we have studied the OZI allowed strong decays of the low-lying $1 P$ - and $1 D$-wave $\Omega$ baryons within the ${ }^{3} P_{0}$ model. It is found that $\Omega(2012)$ is most likely to be a $1 P$-wave $\Omega$ state with $J^{P}=3 / 2^{-}$. We also find that the $\Omega(2250)$ state could be assigned as a $1 D$-wave state with $J^{P}=5 / 2^{+}$. The other missing $1 P$ - and $1 D$-wave $\Omega$ baryons may have large potentials to be observed in their main decay channels.
\end{abstract}

DOI: 10.1103/PhysRevD.98.114023

\section{INTRODUCTION}

The study of the hadron spectrum is an important way for us to understand strong interactions. For the baryon spectra, the classification based on $S U(3)_{f}$ flavor symmetry has been achieved a great success. The $\Omega$ hyperon as a member of baryon decuplet in the quark model was unambiguously discovered in both production and decay at BNL about one half century ago [1]. More excited $\Omega$ baryons should exist as well according to $S U(6) \times O(3)$ symmetry. In theory, the mass spectrum of $\Omega$ hyperon has been predicted within many models, such as the Skyrme model [2], various constituent quark models [3-12], the lattice gauge theory $[13,14]$, and so on. However, in experiments there are only a few information of the excited $\Omega$ baryons. In the review of particle physics from the Particle Data Group (PDG), except for the ground state $\Omega(1672)$, only three possible excited $\Omega$ baryons are listed: $\Omega(2250), \Omega(2380)$, and $\Omega(2470)$ [15]. Their nature is still rather uncertain with three- or two-star ratings. Fortunately, the Belle II experiments offer a great opportunity for our study of the $\Omega$ spectrum.

\footnotetext{
*guilongcheng@hunnu.edu.cn

†vqifang@hunnu.edu.cn

zhongxh@hunnu.edu.cn
}

Published by the American Physical Society under the terms of the Creative Commons Attribution 4.0 International license. Further distribution of this work must maintain attribution to the author(s) and the published article's title, journal citation, and DOI. Funded by SCOAP ${ }^{3}$.
Very recently, a candidate of excited $\Omega$ baryon, $\Omega(2012)$, was observed by the Belle II collaboration [16]. The measured mass and width are

$$
\begin{aligned}
M & =2012.4 \pm 0.7(\text { stat }) \pm 0.6(\text { syst }) \mathrm{MeV}, \\
\Gamma & =6.4_{-2.0}^{+2.5}(\text { stat }) \pm 1.6(\text { syst }) \mathrm{MeV}
\end{aligned}
$$

respectively. In various quark models [3-12], the masses of the first orbital $(1 P)$ excitations of $\Omega$ states are predicted to be $\sim 2.0 \mathrm{GeV}$. The newly observed state $\Omega(2012)$ may be a good candidate of the $1 P$-wave $\Omega$ state. Recently, to study the possible interpretation of $\Omega(2012)$, its strong decays were calculated with the chiral quark model [17], where it was shown that $\Omega(2012)$ could be assigned to the spin-parity $J^{P}=3 / 2^{-} 1 P$-wave state. However, the spinparity $J^{P}=1 / 2^{-}$state can't be completely ruled out. Furthermore, the mass and strong decay patten of $\Omega(2012)$ also were studied by QCD sum rule $[18,19]$. As its mass is very close to $\Xi(1530) K$ threshold, there is also some work that considered it as a $J^{P}=3 / 2^{-} K \Xi^{*}$ molecular state [20-22], or a dynamically generated state [23]. In Ref. [24], by a flavor $S U(3)$ analysis the authors suggested $\Omega(2012)$ to be a number of $3 / 2^{-}$decuplet baryon if the sum of branching ratios for the decay $\Omega(2012) \rightarrow \Xi \bar{K} \pi, \Omega^{-} \pi \pi$ is not too large $(\leq 70 \%)$.

In the present work, to further reveal the nature of $\Omega(2012)$ and better understand the properties of the excited $\Omega$ states, we study the Okubo-Zweig-Iizuka (OZI) allowed two-body strong decays of the $1 P$ - and $1 D$-wave baryons 
TABLE I. The theory predicted masses (MeV) and spin-flavor-space wave functions of $\Omega$ baryons under $S U(6)$ quark model classification are listed below. We denote the baryon states as $\left|\mathrm{N}_{6},{ }^{2 S+1} \mathrm{~N}_{3}, N, L, J^{P}\right\rangle$ where $N_{6}$ stands for the irreducible representation of spin-flavor $S U(6)$ group, $N_{3}$ stands for the irreducible representation of flavor $S U(3)$ group and $\mathrm{N}, \mathrm{L}, J^{P}$ as principal quantum number, total orbital angular momentum and spin-parity, respectively [17]. The $\phi, \chi, \psi$ denote flavor, spin, and spatial wave function, respectively. The Clebsch-Gorden coefficients of spin-orbital coupling have been omitted.

\begin{tabular}{|c|c|c|c|c|c|c|c|}
\hline & \multirow[b]{2}{*}{ States } & \multirow[b]{2}{*}{ Wave function } & \multirow[b]{2}{*}{ [4] } & \multicolumn{3}{|c|}{ Theory } & \multirow[b]{2}{*}{ [13] } \\
\hline & & & & [3] & [2] & [7] & \\
\hline $1 \mathrm{~S}$ & $\left|56,{ }^{4} 10,0,0,3 / 2^{+}\right\rangle$ & $\phi^{s} \chi^{s} \psi_{000}^{s}$ & 1678 & 1635 & 1694 & 1675 & $1642(17)$ \\
\hline \multirow[t]{2}{*}{$1 \mathrm{P}$} & $\left|70,{ }^{2} 10,1,1,1 / 2^{-}\right\rangle$ & $\frac{1}{\sqrt{2}}\left(\phi^{s} \chi^{\rho} \psi_{11 L_{z}}^{\rho}+\phi^{s} \chi^{\lambda} \psi_{11 L_{z}}^{\lambda}\right)$ & 1941 & 1950 & 1837 & 2020 & 1944(56) \\
\hline & $\left|70,{ }^{2} 10,1,1,3 / 2^{-}\right\rangle$ & $\frac{1}{\sqrt{2}}\left(\phi^{s} \chi^{\rho} \psi_{11 L_{z}}^{\rho}+\phi^{s} \chi^{\lambda} \psi_{11 L_{z}}^{\lambda}\right)$ & 2038 & 2000 & 1978 & 2020 & $2049(32)$ \\
\hline \multirow[t]{6}{*}{$1 \mathrm{D}$} & $\left|56,{ }^{4} 10,2,2,1 / 2^{+}\right\rangle$ & $\phi^{s} \chi^{s} \psi_{22 L_{z}}^{s}$ & 2301 & 2255 & 2140 & 2210 & $2350(63) / 2481(51)$ \\
\hline & $\left|56,{ }^{4} 10,2,2,3 / 2^{+}\right\rangle$ & $\phi^{s} \chi^{s} \psi_{22 L_{z}}^{s}$ & $2173 / 2304$ & 2280 & 2282 & 2215 & \multirow{5}{*}{ 2470(49) } \\
\hline & $\left|70,{ }^{2} 10,2,2,3 / 2^{+}\right\rangle$ & $\frac{1}{\sqrt{2}}\left(\phi^{s} \chi^{\rho} \psi_{22 L_{z}}^{\rho}+\phi^{s} \chi^{\lambda} \psi_{22 L_{z}}^{\lambda}\right)$ & $2173 / 2304$ & 2345 & 2282 & 2265 & \\
\hline & $\left|56,{ }^{4} 10,2,2,5 / 2^{+}\right\rangle$ & $\phi^{s} \chi^{s} \psi_{22 L_{z}}^{s}$ & 2401 & 2280 & $\cdots$ & 2225 & \\
\hline & $\left|70,{ }^{2} 10,2,2,5 / 2^{+}\right\rangle$ & $\frac{1}{\sqrt{2}}\left(\phi^{s} \chi^{\rho} \psi_{22 L_{z}}^{\rho}+\phi^{s} \chi^{\lambda} \psi_{22 L_{z}}^{\lambda}\right)$ & 2401 & 2345 & $\cdots$ & 2265 & \\
\hline & $\left|56,{ }^{4} 10,2,2,7 / 2^{+}\right\rangle$ & $\phi^{s} \chi^{s} \psi_{22 L_{z}}^{s}$ & 2332 & 2295 & $\cdots$ & 2210 & \\
\hline
\end{tabular}

with the widely used ${ }^{3} P_{0}$ model. The quark model classification for the $1 P$ - and $1 D$-wave $\Omega$ baryons and their theoretical masses are listed in Table I. The spatial wave functions for the $\Omega$ baryons are described by harmonic oscillators. According to our calculations, we find that (i) the newly observed $\Omega(2012)$ resonance is most likely to be the $1 P$-wave $\Omega$ state with spin-parity $J^{P}=$ $3 / 2^{-}$and the experimental data can be reasonably described. The other $1 P$-wave state with $J^{P}=1 / 2^{-}$might be broader state with a width of dozens of $\mathrm{MeV}$. The ${ }^{3} P_{0}$ results are consistent with the recent predictions of the chiral quark model [17]. (ii) The $\Omega(2250)$ resonance listed in PDG may be a good candidate of the $J^{P}=5 / 2^{+} 1 D$ wave state $\left|56,{ }^{4} 10,2,2,5 / 2^{+}\right\rangle$or $\left|70,{ }^{2} 10,2,2,5 / 2^{+}\right\rangle$. Although the widths of the $D$-wave states predicted within the ${ }^{3} P_{0}$ model are systematically larger that those predicted with the chiral quark model [17], these states may be observed in their main decay channels in future experiments for their relatively narrow width.

This paper is organized as follows. First, we give a brief review of the ${ }^{3} P_{0}$ model in Sec. II. Second, we present the numerical results of strong decay of $1 P$ - and $1 D$-wave $\Omega$ baryon in Sec. III. Finally, a summary of our results is given in Sec. IV.

\section{THE ${ }^{3} P_{0}$ MODEL}

The ${ }^{3} P_{0}$ model, is also called the quark pair creation (QPC) model. It was first proposed by Micu [25], Carlitz and Kislinger [26], and developed by the Orsay group and Yaouanc et al. [27-32]. In the model, it assumes that a pair of quarks $q \bar{q}$ is created from the vacuum with $J^{P C}=$ $0^{++}\left({ }^{2 S+1} L_{J}={ }^{3} P_{0}\right)$ when the initial hadron A decays, and the quarks from the hadron $A$ regroups with the created quarks form two daughter hadrons B and C. For baryon decays, two quarks of the initial baryon regroup with the created quark to form a daughter baryon, and the remaining one quark regroup with the created antiquark to form a meson. The process of baryon decays is shown in Fig. 1.

The transition operator under the ${ }^{3} P_{0}$ model is written as

$$
\begin{aligned}
T= & -3 \gamma \sum_{m}\langle 1 m ; 1-m \mid 00\rangle \int d^{3} \vec{p}_{4} d^{3} \vec{p}_{5} \delta^{3}\left(\vec{p}_{4}+\vec{p}_{5}\right) \\
& \times \mathcal{Y}_{1}^{m}\left(\frac{\vec{p}_{4}-\vec{p}_{5}}{2}\right) \chi_{1,-m}^{45} \varphi_{0}^{45} \omega_{0}^{45} b_{4 i}^{\dagger}\left(\vec{p}_{4}\right) d_{5 j}^{\dagger}\left(\vec{p}_{5}\right),
\end{aligned}
$$

where the pair-strength $\gamma$ is a dimensionless parameter, $i$ and $j$ are the color indices of the created quark $q_{4}$ and antiquark $\bar{q}_{5} \cdot \varphi_{0}^{45}=(u \bar{u}+d \bar{d}+s \bar{s}) / \sqrt{3}$ and $\omega_{0}^{45}=\delta_{i j}$ stand for the flavor and color singlet, respectively. $\chi_{1,-m}^{45}$ is the spin triplet state and $\mathcal{Y}_{1}^{m}(\vec{p}) \equiv|\vec{p}| Y_{1}^{m}\left(\theta_{p}, \phi_{p}\right)$ is a solid harmonic polynomial corresponding to the $P$-wave quark pair. $b_{4 i}^{\dagger}\left(\vec{p}_{4}\right) d_{5 j}^{\dagger}\left(\vec{p}_{5}\right)$ denotes the creation operator in the vacuum.

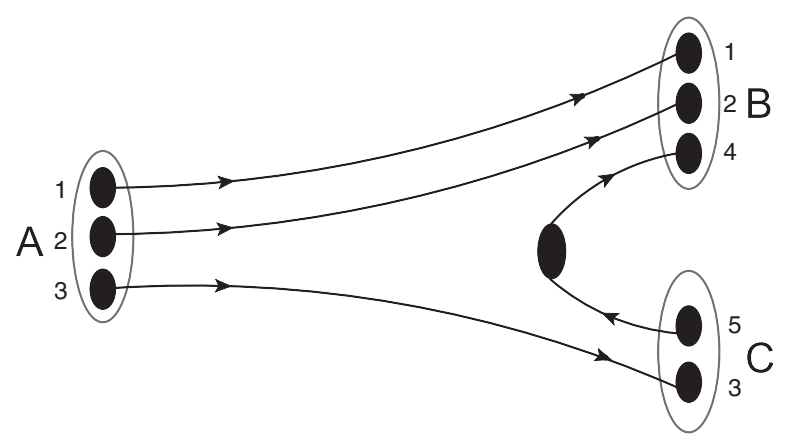

FIG. 1. The decay process of $A \rightarrow B+C$ in the ${ }^{3} P_{0}$ model. 
According to the definition of the mock state [11], the baryons $\mathrm{A}$ and $\mathrm{B}$, and meson $\mathrm{C}$ are defined as follows:

$$
\begin{aligned}
\mid A & \left.\left(n_{A}^{2 S_{A}+1} L_{A\left(J_{A}, M_{J_{A}}\right.}\right)\left(\vec{P}_{A}\right)\right\rangle \\
= & \sqrt{2 E_{A}} \sum_{M_{L_{A}}, M_{S_{A}}}\left\langle L_{A} M_{L_{A}} ; S_{A} M_{S_{A}} \mid J_{A} M_{J_{A}}\right\rangle \\
& \times \int \delta^{3}\left(\vec{p}_{1}+\vec{p}_{2}+\vec{p}_{3}-\vec{P}_{A}\right) d^{3} \vec{p}_{1} d^{3} \vec{p}_{2} d^{3} \vec{p}_{3} \\
& \times \Psi_{n_{A} L_{A} M_{L_{A}}}\left(\vec{p}_{1}, \vec{p}_{2}, \vec{p}_{3}\right) \chi_{S_{A} M_{S_{A}}}^{123} \varphi_{A}^{123} \omega_{A}^{123} \\
& \times\left|q_{1}\left(\vec{p}_{1}\right) q_{2}\left(\vec{p}_{2}\right) q_{3}\left(\vec{p}_{3}\right)\right\rangle, \\
\mid B & \left.\left(n_{B}^{2 S_{B}+1} L_{B\left(J_{B} M_{J_{B}}\right)}\right)\left(\vec{P}_{B}\right)\right\rangle \\
= & \sqrt{2 E_{B}} \sum_{M_{L_{B}} M_{S_{B}}}\left\langle L_{B} M_{L_{B}} ; S_{B} M_{S_{B}} \mid J_{B} M_{J_{B}}\right\rangle \\
& \times \int \delta^{3}\left(\vec{p}_{1}+\vec{p}_{2}+\vec{p}_{4}-\vec{P}_{B}\right) d^{3} \vec{p}_{1} d^{3} \vec{p}_{2} d^{3} \vec{p}_{4} \\
& \times \Psi_{n_{B} L_{B} M_{L_{B}}}\left(\vec{p}_{1}, \vec{p}_{2}, \vec{p}_{4}\right) \chi_{S_{B} M_{S_{B}}}^{124} \varphi_{B}^{124} \omega_{B}^{124} \\
& \times\left|q_{1}\left(\vec{p}_{1}\right) q_{2}\left(\vec{p}_{2}\right) q_{4}\left(\vec{p}_{4}\right)\right\rangle,
\end{aligned}
$$

$$
\begin{aligned}
& \left|C\left(n_{C}^{2 S_{C}+1} L_{C\left(J_{C}, M_{J_{C}}\right)}\right)\left(\vec{P}_{C}\right)\right\rangle \\
& =\sqrt{2 E_{C}} \sum_{M_{L_{C}}, M_{S_{C}}}\left\langle L_{C} M_{L_{C}} ; S_{C} M_{S_{C}} \mid J_{C} M_{J_{C}}\right\rangle \\
& \quad \times \int \delta^{3}\left(\vec{p}_{3}+\vec{p}_{5}-\vec{P}_{C}\right) d^{3} \vec{p}_{3} d^{3} \vec{p}_{5} \\
& \quad \times \Psi_{n_{C} L_{C} M_{L_{C}}}\left(\vec{p}_{3}, \vec{p}_{5}\right) \chi_{S_{C} M_{S_{C}}}^{35} \\
& \quad \times \varphi_{C}^{35} \omega_{C}^{35}\left|q_{3}\left(\vec{p}_{3}\right) q_{5}\left(\vec{p}_{5}\right)\right\rangle,
\end{aligned}
$$

where the subscripts $1,2,3$ denote the quarks of the initial baryon $A .1,2$, and 4 denote the quarks of the final baryon $B, 3$ and 5 stand for the quark and antiquark of the final meson $C$, respectively. $\vec{p}_{i}(i=1,2,3)$ are the momentum of quarks in baryon $A . \vec{p}_{i}(i=1,2,4)$ are the momentum of quarks in baryon $B . \vec{p}_{3}$ and $\vec{p}_{5}$ are the momentum of the quark and antiquark in meson $C . \vec{P}_{A}, \vec{P}_{B}, \vec{P}_{C}$ denotes the momentum of state A, B, C. $S_{A}, S_{B}, S_{C}$ and $J_{A}, J_{B}, J_{C}$ represent the total spin and the total angular momentum of state A, B, C. $\Psi_{n_{A} L_{A} M_{L_{A}}}, \Psi_{n_{A} L_{A} M_{L_{A}}}$, and $\Psi_{n_{C} L_{C} M_{L_{C}}}$ denotes the spatial wave functions of the baryon and meson in momentum space, respectively.

The decay amplitude of the $\Omega$ baryon in ${ }^{3} P_{0}$ is written as

$$
\begin{aligned}
& \mathcal{M}^{M_{J_{A}} M_{J_{B}} M_{J_{C}}}=\left\langle B\left(n_{B}^{2 S_{B}+1} L_{B\left(J_{B}, M_{J_{B}}\right)}\right), C\left(n_{C}^{2 S_{C}+1} L_{C\left(J_{C}, M_{J_{C}}\right)}\right)|T| A\left(n_{A}^{2 S_{A}+1} L_{A\left(J_{A}, M_{J_{A}}\right)}\right\rangle\right. \\
& =-3 \gamma \sqrt{8 E_{A} E_{B} E_{C}} \sum_{M_{L_{A}}, M_{S_{A}}} \sum_{M_{L_{B}}, M_{S_{B}}} \sum_{M_{L_{C}}, M_{S_{C}}, m}\left\langle L_{A} M_{L_{A}} ; S_{A} M_{S_{A}} \mid J_{A} M_{J_{A}}\right\rangle\langle 1 m ; 1-m \mid 00\rangle \\
& \times\left\langle L_{B} M_{L_{B}} ; S_{B} M_{S_{B}} \mid J_{B} M_{J_{B}}\right\rangle\left\langle L_{C} M_{L_{C}} ; S_{C} M_{S_{C}} \mid J_{C} M_{J_{C}}\right\rangle\left\langle\chi_{S_{B} M_{S_{B}}}^{124} \chi_{S_{C} M_{S_{C}}}^{35} \mid \chi_{S_{A} M_{S_{A}}}^{123} \chi_{1-m}^{45}\right\rangle
\end{aligned}
$$

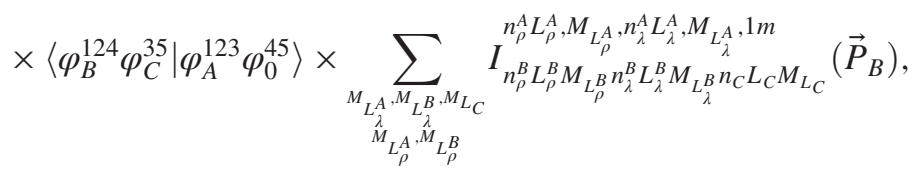

$$
\begin{aligned}
& \left\langle\chi_{S_{B} M_{S_{B}}}^{124} \chi_{S_{C} M_{S_{C}}}^{35} \mid \chi_{S_{A} M_{S_{A}}}^{123} \chi_{1-m}^{45}\right\rangle=\left\langle S_{1} M_{S_{1}} ; S_{2} M_{S_{2}} \mid S_{12} M_{S_{12}}\right\rangle\left\langle S_{12} M_{S_{12}} ; S_{3} M_{S_{3}} \mid S_{A} M_{S_{A}}\right\rangle \\
& \times\left\langle S_{1} M_{S_{1}} ; S_{2} M_{S_{2}} \mid S_{12} M_{S_{12}}\right\rangle\left\langle S_{12} M_{S_{12}} ; S_{4} M_{S_{4}} \mid S_{B} M_{S_{B}}\right\rangle \\
& \times\left\langle S_{3} M_{S_{3}} ; S_{5} M_{S_{5}} \mid S_{C} M_{S_{C}}\right\rangle\left\langle S_{4} M_{S_{4}} ; S_{5} M_{S_{5}} \mid 10\right\rangle .
\end{aligned}
$$

In the Eq. (5), $\left\langle\chi_{S_{B} M_{S_{B}}}^{124} \chi_{S_{C} M_{S_{C}}}^{35} \mid \chi_{S_{A} M_{S_{A}}}^{123} \chi_{1-m}^{45}\right\rangle$ and $\left\langle\varphi_{B}^{124} \varphi_{C}^{35} \mid \varphi_{A}^{123} \varphi_{0}^{45}\right\rangle$ stand for the spin matrix and the flavor matrix, respectively. $S_{i}$ stand for the spin of $i$ th quark and $S_{12}$ stand for the total spin of 1 and 2 quarks. The prefactor 3 in front of $\gamma$ arises from the fact that the three decay processes of the $\Omega$ baryon in ${ }^{3} P_{0}$ model are equivalent. The summed magnetic quantum numbers are not completely independent of each other.

The overlap integral in the momentum space is written as

$$
\begin{aligned}
I_{n_{\rho}^{B} L_{\rho}^{B} M_{L_{\rho}^{B}}^{B} n_{\lambda}^{B} L_{\lambda}^{B} M_{L_{\lambda}^{B_{\lambda}}}^{A}, M_{C} L_{C} M_{L_{C}}^{A} h_{B}^{A}, M_{L^{A}}, 1 m}\left(\vec{P}_{B}\right)= & \int d^{3} \vec{p}_{1} d^{3} \vec{p}_{2} d^{3} \vec{p}_{3} d^{3} \vec{p}_{4} d^{3} \vec{p}_{5} \delta^{3}\left(\vec{p}_{1}+\vec{p}_{2}+\vec{p}_{3}-\vec{P}_{A}\right) \delta^{3}\left(\vec{p}_{4}+\vec{p}_{5}\right) \\
& \times \delta^{3}\left(\vec{p}_{1}+\vec{p}_{2}+\vec{p}_{4}-\vec{P}_{B}\right) \delta^{3}\left(\vec{p}_{3}+\vec{p}_{5}-\vec{P}_{C}\right) \Psi_{A}\left(\vec{p}_{1}, \vec{p}_{2}, \vec{p}_{3}\right) \Psi_{B}^{*}\left(\vec{p}_{1}, \vec{p}_{2}, \vec{p}_{4}\right) \Psi_{C}^{*}\left(\vec{p}_{3}, \vec{p}_{5}\right) \\
= & \int d \vec{p}_{\rho} d \vec{p}_{\lambda}|J| \Psi_{A}\left(\vec{p}_{\lambda}^{A}, \vec{p}_{\rho}^{A}\right) \Psi_{B}\left(\vec{P}_{B}, \vec{p}_{\lambda}^{A}, \vec{p}_{\rho}^{A}\right) \Psi_{C}\left(-\vec{P}_{B}, \vec{p}_{\lambda}^{A}, \vec{p}_{\rho}^{A}\right)
\end{aligned}
$$




$$
\begin{aligned}
& =\int d \vec{p}_{\rho}^{A} d \vec{p}_{\lambda}^{A} d \Omega_{\rho}^{A} d \Omega_{\lambda}^{A}|J| \phi_{n_{\rho}^{A} L_{\rho}^{A}}\left(\vec{p}_{\rho}^{A}\right) Y_{L_{\rho}^{A} M_{L_{\rho}^{A}}}\left(\Omega_{\rho}^{A}\right) \phi_{n_{\lambda}^{A} L_{\lambda}^{A}}\left(\vec{p}_{\lambda}^{A}\right) Y_{L_{\lambda}^{A} M_{L_{\lambda}^{A}}}\left(\Omega_{\lambda}^{A}\right) \\
& \times \phi_{n_{\rho}^{B} L_{\rho}^{B}}\left(\vec{p}_{\rho}^{A}\right) Y_{L_{\rho}^{B} M_{L_{\rho}^{B}}}\left(\Omega_{\rho}^{B}\right) \phi_{n_{\lambda}^{B} L_{\lambda}^{B}}\left(\vec{p}_{\lambda}^{A}-\sqrt{\frac{3}{2}} \frac{2 m_{3}}{2 m_{3}+m_{q}} \vec{P}_{B}\right) Y_{L_{\lambda}^{B} M_{L_{\lambda}^{B}}}\left(\Omega_{\lambda}^{B}\right) \\
& \times \phi_{n_{C} L_{C}}\left(\frac{m_{3}}{m_{3}+m_{q}} \vec{P}_{B}-\sqrt{\frac{2}{3}} \vec{p}_{\lambda}^{A}\right) Y_{L_{C} M_{L_{C}}}\left(\Omega^{C}\right) \mathcal{Y}_{1}^{m}\left(\vec{P}_{B}-\sqrt{\frac{2}{3}} \vec{p}_{\lambda}^{A}\right),
\end{aligned}
$$

where $\vec{p}_{\rho}=\frac{1}{\sqrt{2}}\left(\frac{m_{2} \vec{p}_{1}-m_{1} \vec{p}_{2}}{m_{1}+m_{2}}\right)$ and $\vec{p}_{\lambda}=\sqrt{\frac{2}{3}}\left(\frac{m_{3}\left(\vec{p}_{1}+\vec{p}_{2}\right)-\left(m_{1}+m_{2}\right) \vec{p}_{3}}{m_{1}+m_{2}+m_{3}}\right)$ stand for the momentum corresponding to $\rho$ and $\lambda$ Jacobi coordinates in the center mass frame of baryon A, and $|J|$ stand for the Jacobi determinant which determined by the definition of $p_{\rho}, p_{\lambda} . m_{i}$ denote the mass of $i$ th quark, and $m_{q}$ denote the mass of the created quark pairs. The $n_{\rho}$ and $L_{\rho}$ denote the nodal and orbital angular momentum between the 1, 2 quarks (see Fig. 1), while the $n_{\lambda}$ and $L_{\lambda}$ stand for the nodal and orbital angular momentum between the 1, 2 quarks system and the 3 quark (see Fig. 1).

In the calculations, simple harmonic oscillator (SHO) wave functions is employed as the hadron wave function. The momentum space wave function of baryon is

$$
\begin{aligned}
& \Psi_{A}\left(\vec{P}_{A}\right)=N \psi_{n_{\rho_{A}} l_{\rho_{A}} M_{l_{\rho_{A}}}}\left(\vec{p}_{\rho_{A}}\right) \psi_{n_{\lambda_{A}} l_{\lambda_{A}} M_{l_{\lambda_{A}}}}\left(\vec{p}_{\lambda_{A}}\right) \\
& \psi_{n l M_{l}}(\vec{p})=(-1)^{n}(-i)^{l}\left[\frac{2^{l+2}}{\sqrt{\pi}(2 l+1) ! !}\right]^{\frac{1}{2}} \\
& \times\left(\frac{1}{\beta}\right)^{l+\frac{3}{2}} \exp \left(-\frac{\vec{p}^{2}}{2 \beta^{2}}\right) L_{n}^{l+1 / 2}\left(\frac{\vec{p}^{2}}{\beta^{2}}\right) \mathcal{Y}_{l}^{m}(\vec{p}),
\end{aligned}
$$

where $N$ stands for a normalization coefficient of the wave function and $L_{n}^{l+1 / 2}\left(\frac{\vec{p}^{2}}{\beta^{2}}\right)$ is the Laguerre polynomial function. The Clebsch-Gorden coefficients of $l_{\rho}, l_{\lambda}$ coupling are equal to 1 in our case.

The ground state wave function of a meson in the momentum space is

$$
\Psi_{0,0}=\left[\frac{R^{2}}{\pi}\right]^{\frac{3}{4}} \exp \left(-\frac{R^{2} \vec{p}_{a b}^{2}}{2}\right)
$$

where the $\vec{p}_{a b}$ stands for the relative momentum between the quark and antiquark in the meson. As all hadrons in the final states are $S$-wave in this work, Eq. (7) can be further expressed as follows

$\Pi\left(L_{\rho}^{A}, M_{L_{\rho}^{A}}, L_{\lambda}^{A}, M_{L_{\lambda}^{A}}, m\right) \equiv I_{n_{\rho}^{B} L_{\rho}^{B} M_{L_{\rho}^{B}} n_{\lambda}^{B} L_{\lambda}^{B} M_{L_{\lambda}^{B}} n_{C} L_{C} M_{L_{C}}^{A}, M_{L^{A}}, n_{\lambda}^{A} L_{\lambda}^{A}, M_{L_{A}^{A}}, 1 m}$ the expressions of $\Pi\left(L_{\rho_{A}}, M_{L_{\rho}^{A}}, L_{\lambda_{A}}, M_{L_{\lambda}^{A}}, m\right)$ and harmonic oscillator wave function for the $S$-wave, $P$-wave, $D$-wave $\Omega$ baryons are collected in the Appendix A and B.

The decay width $\Gamma$ of the process $A \rightarrow B+C$ is

$$
\Gamma=\pi^{2} \frac{|\vec{p}|}{m_{A}^{2}} \frac{1}{2 J_{A}+1} \sum_{M_{J_{A}}, M_{J_{B}}, M_{J_{C}}} \mid \mathcal{M}^{\left.M_{J_{A}} M_{J_{B}} M_{J_{C}}\right|^{2}},
$$

where $J_{A}$ are the total angular momentum of the initial baryon $A$. $\vec{p}$ is the momentum of the final baryon in the center of mass frame of the initial baryon $A$

$|\vec{p}|=\frac{\sqrt{\left[m_{A}^{2}-\left(m_{B}-m_{C}\right)^{2}\right]\left[m_{A}^{2}-\left(m_{B}+m_{C}\right)^{2}\right]}}{2 m_{A}}$,

where $m_{A}, m_{B}$, and $m_{C}$ are the mass of the initial and final hadrons.

In order to partly remedy the inadequacy of the nonrelativistic wave function as the relative momentum $\vec{p}$ increases [33-37], the decay amplitude is written as

$$
\mathcal{M}(\vec{p}) \rightarrow \gamma_{f} \mathcal{M}\left(\gamma_{f} \vec{p}\right),
$$

where $\gamma_{f}$ denotes a commonly Lorentz boost factor, $\gamma_{f}=m_{B} / E_{B}$. In most decays, the three momenta carried by the final state baryons are relatively small, which means the nonrelativistic prescription is reasonable and the corrections from the Lorentz boost is not drastic.

\section{NUMERICAL RESULTS AND ANALYSIS}

In our calculations, we adopt $m_{u}=m_{d}=350 \mathrm{MeV}$, $m_{s}=450 \mathrm{MeV}$ for the constituent quark masses. The masses of the $\Omega$ baryons listed in Table I, the masses of $K$ mesons and $\Xi$ baryons are taken from the PDG [15]. The quantum numbers involved in the calculations are listed in Table II. Due to the orthogonal relationship of the wave functions, only the $\lambda$ excited mode contributes. There are three harmonic oscillator parameters, the $\beta_{\rho}$ and $\beta_{\lambda}$ and $R$ in baryon and meson wave functions, respectively. We adopt $R=2.5 \mathrm{GeV}^{-1}$ for $K$ mesons [38]. The parameter $\beta_{\rho}$ of the $\rho$-mode excitation between the 1, 2 quarks (see Fig. 1) is taken as $\beta_{\rho}=0.4 \mathrm{GeV}$ [39]. The $\beta_{\lambda}$ is obtained with the relation [40]: 
TABLE II. The Quantum numbers of $1 P$-wave and $1 D$-wave $\Omega$ baryons. Due to the orthogonal relationship of the wave functions, only the $\lambda$ excited mode contributes.

\begin{tabular}{lcccccccc}
\hline \hline States & $J$ & $n_{\rho}$ & $l_{\rho}$ & $n_{\lambda}$ & $l_{\lambda}$ & $L$ & $S_{\rho}$ & $S$ \\
\hline$\left|70,{ }^{2} 10,1,1,1 / 2^{-}\right\rangle$ & $\frac{1}{2}$ & 0 & 0 & 0 & 1 & 1 & 1 & $\frac{1}{2}$ \\
$\left|70,{ }^{2} 10,1,1,3 / 2^{-}\right\rangle$ & $\frac{3}{2}$ & 0 & 0 & 0 & 1 & 1 & 1 & $\frac{1}{2}$ \\
$\left|56,{ }^{4} 10,2,2,1 / 2^{+}\right\rangle$ & $\frac{1}{2}$ & 0 & 0 & 0 & 2 & 2 & 1 & $\frac{3}{2}$ \\
$\left|56,{ }^{4} 10,2,2,3 / 2^{+}\right\rangle$ & $\frac{3}{2}$ & 0 & 0 & 0 & 2 & 2 & 1 & $\frac{3}{2}$ \\
$\left|70,{ }^{2} 10,2,2,3 / 2^{+}\right\rangle$ & $\frac{3}{2}$ & 0 & 0 & 0 & 2 & 2 & 1 & $\frac{1}{2}$ \\
$\left|56,{ }^{4} 10,2,2,5 / 2^{+}\right\rangle$ & $\frac{5}{2}$ & 0 & 0 & 0 & 2 & 2 & 1 & $\frac{3}{2}$ \\
$\left|70,{ }^{2} 10,2,2,5 / 2^{+}\right\rangle$ & $\frac{5}{2}$ & 0 & 0 & 0 & 2 & 2 & 1 & $\frac{1}{2}$ \\
$\left|56,{ }^{4} 10,2,2,7 / 2^{+}\right\rangle$ & $\frac{7}{2}$ & 0 & 0 & 0 & 2 & 2 & 1 & $\frac{3}{2}$ \\
\hline \hline
\end{tabular}

$$
\beta_{\lambda}=\left(\frac{3 m_{3}}{2 m_{1}+m_{3}}\right)^{\frac{1}{4}} \beta_{\rho}
$$

For the quark pair creation strength from the vacuum, we take as those in Ref. [38], $\gamma=6.95$.

\section{A. The $1 P$-wave states}

According to the $S U(6)$ supermultiplet classification (see Table I), there are two $1 P$-wave states with $J^{P}=\frac{1}{2}^{-}$and $J^{P}=\frac{3}{2}$, i.e., $\left|70,{ }^{2} 10,1,1,1 / 2^{-}\right\rangle$and $\left|70,{ }^{2} 10,1,1,3 / 2^{-}\right\rangle$. The mass of the newly observed $\Omega(2012)$ state is close to the $1 P$-wave $\Omega$ baryon predicted in various quark models (see Table I). Assuming $\Omega(2012)$ as a candidate of the $1 P$-wave $\Omega$ baryons, we calculate the OZI-allowed two body strong decays in the ${ }^{3} P_{0}$ model, and list our results in Table III.

It is found that if one assigns $\Omega(2012)$ as the $J^{P}=\frac{1}{2}^{-}$ state $\left|70,{ }^{2} 10,1,1,1 / 2^{-}\right\rangle$, the width is predicted to be

$$
\Gamma_{\text {total }}^{\text {th }} \simeq 43 \mathrm{MeV}
$$

which is too large to be comparable with the width of $\Omega(2012)$. This width predicted in the ${ }^{3} P_{0}$ model is about a factor 3 larger than that predicted within the chiral quark model [17]. As the mass of $\frac{1}{2}-\Omega$ baryon are predicted about $1.95 \mathrm{GeV}$ in some models $[3,4,13]$. In Fig. 2 we shows its strong decay properties as functions of mass in range of

TABLE III. The total decay widths $(\mathrm{MeV})$ of the $\mid 70,{ }^{2} 10,1,1$, $\left.1 / 2^{-}\right\rangle$and $\left|70,{ }^{2} 10,1,1,3 / 2^{-}\right\rangle$states with mass $M=2012 \mathrm{MeV}$. $\Gamma_{\text {total }}^{\text {th }}$ denotes the total decay width and $\mathfrak{B}$ stands for the radio of the branching fraction $\Gamma\left[\Xi^{0} K^{-}\right] / \Gamma\left[\Xi^{-} \bar{K}^{0}\right]$. The results of Ref. [17] are also listed for a comparison. The units of widths is $\mathrm{MeV}$.

\begin{tabular}{lcccc}
\hline \hline States & $\Gamma_{\text {total }}^{\text {th }}$ & $\Gamma_{\text {total }}^{\text {th }}[17]$ & $\mathfrak{B}$ & $\mathfrak{B}[17]$ \\
\hline$\left|70,{ }^{2} 10,1,1,1 / 2^{-}\right\rangle$ & 43.0 & 15.2 & 0.96 & 0.95 \\
$\left|70,{ }^{2} 10,1,1,3 / 2^{-}\right\rangle$ & 8.19 & 6.64 & 1.11 & 1.12 \\
\hline \hline
\end{tabular}

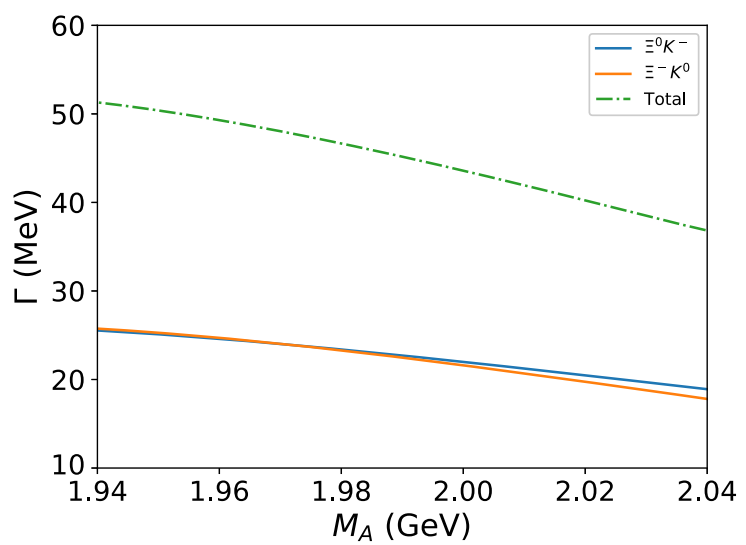

FIG. 2. The decay width of the $J^{P}=\frac{1}{2}^{-} \Omega$ baryon as functions of the initial state mass.

1.94-2.04 GeV. It is found that the decay width of $\frac{1-}{2} \Omega$ baryon is about $\Gamma \sim 40-50 \mathrm{MeV}$ and dominantly decays to $\Xi K$ channel. As future experimental statistics increases, it is possible to find the $\frac{1-}{2} \Omega$ baryon in this channel.

On the other hand, assigning $\Omega(2012)$ as the $J^{P}=\frac{3}{2}^{-}$ state $\left|70,{ }^{2} 10,1,1,3 / 2^{-}\right\rangle$, we find that the width

$$
\Gamma_{\text {total }}^{\text {th }} \simeq 8 \mathrm{MeV},
$$

and the branching fraction ratio

$$
R^{\text {th }}=\frac{\Gamma\left[\left|70,{ }^{2} 10,1,1,3 / 2^{-}\right\rangle \rightarrow \Xi^{0} K^{-}\right]}{\Gamma\left[\left|70,{ }^{2} 10,1,1,3 / 2^{-}\right\rangle \rightarrow \Xi^{-} \bar{K}^{0}\right]} \simeq 1.1,
$$

are consistent with the measured width $\Gamma^{\exp }=$ $6.4_{-2.0}^{+2.5}$ (stat) \pm 1.6 (syst) and ratio $R^{\exp }=1.2 \pm 0.3$ for $\Omega(2012)$. These ${ }^{3} P_{0}$ model predictions are compatible with those predicted within the chiral quark model [17].

\section{B. The $1 D$-wave states}

According to the quark model classification, there are six $1 D$-wave states. Their masses are predicted to be in the range of 2.2-2.3 GeV in various models (see Table I). Their OZI allowed two-body strong decay channels are $\Xi K$ and $\Xi(1530) K$. With the masses predicted in Ref. [7], we study the strong decay processes of $1 D$-wave states into both $\Xi K$ and $\Xi(1530) K$ channels, and collect their partial decay widths in Table IV.

It is interesting to find that the two $J^{P}=5 / 2^{+}$states $\left|56,{ }^{4} 10,2,2,5 / 2^{+}\right\rangle$and $\left|70,{ }^{2} 10,2,2,5 / 2^{+}\right\rangle$may be good candidates of $\Omega(2250)$ listed in the review book of PDG [15]. (i) The mass of $\Omega(2250)$ is close the predictions of $\left|56,{ }^{4} 10,2,2,5 / 2^{+}\right\rangle$and $\left|70,{ }^{2} 10,2,2,5 / 2^{+}\right\rangle$in the quark model [7]. (ii) The measured width of $\Omega(2250), \Gamma=$ $55 \pm 18 \mathrm{MeV}$, is close to the theoretical predictions, $\Gamma \sim$ $80 / 50 \mathrm{MeV}$ for $\left|56,{ }^{4} 10,2,2,5 / 2^{+}\right\rangle$and $\left|70,{ }^{2} 10,2,2,5 / 2^{+}\right\rangle$, respectively. (iii) The decays modes of $\left|56,{ }^{4} 10,2,2,5 / 2^{+}\right\rangle$ 
TABLE IV. The partial and total decay widths $(\mathrm{MeV})$ of the $1 D$-wave states. $\Gamma_{\text {total }}^{\text {th }}$ denotes the total decay width and $\mathfrak{B}$ stands for the radio of the branching fraction $\Gamma[\Xi K] / \Gamma[\Xi(1530) K]$. The results of Ref. [17] are also shown for a comparison. The widths and masses are in units of $\mathrm{MeV}$.

\begin{tabular}{|c|c|c|c|c|c|c|c|c|c|}
\hline States & Mass [7] & $\Gamma[\Xi K]$ & $\Gamma[\Xi K][17]$ & $\Gamma[\Xi(1530) K]$ & $\Gamma[\Xi(1530) K][17]$ & $\Gamma_{\text {total }}^{\mathrm{th}}$ & $\Gamma_{\text {total }}^{\text {th }}[17]$ & $\mathcal{B}$ & $\mathcal{B}[17]$ \\
\hline$\left|56,{ }^{4} 10,2,2,1 / 2^{+}\right\rangle$ & 2210 & 154 & 51.8 & 16.5 & 4.53 & 171 & 56.3 & 9.36 & 11.4 \\
\hline$\left|56,{ }^{4} 10,2,2,3 / 2^{+}\right\rangle$ & 2215 & 76.8 & 25.8 & 55.4 & 15.7 & 132 & 41.5 & 1.39 & 1.64 \\
\hline$\left|56,{ }^{4} 10,2,2,5 / 2^{+}\right\rangle$ & 2225 & 7.78 & 6.58 & 77.2 & 22.6 & 84.9 & 29.2 & 0.10 & 0.29 \\
\hline$\left|56,{ }^{4} 10,2,2,7 / 2^{+}\right\rangle$ & 2210 & 31.7 & 26.2 & 2.94 & 1.51 & 34.7 & 27.7 & 10.8 & 17.4 \\
\hline$\left|70,{ }^{2} 10,2,2,3 / 2^{+}\right\rangle$ & 2265 & 9.04 & 7.40 & 14.3 & 11.9 & 23.4 & 20.9 & 0.63 & 0.62 \\
\hline$\left|70,{ }^{2} 10,2,2,5 / 2^{+}\right\rangle$ & 2265 & 4.34 & 0.99 & 50.0 & 11.6 & 54.4 & 13.4 & 0.09 & 0.08 \\
\hline
\end{tabular}

and $\left|70,{ }^{2} 10,2,2,5 / 2^{+}\right\rangle$are dominated by $\Xi(1530) K$, which is also consistent with the fact that the $\Omega(2250)$ was seen in the $\Xi(1530) K$ and $\Xi^{-} \pi^{+} K^{-}$channels. In [17], we predicted that $\Omega(2250)$ is more likely to be the $J^{P}=$ $5 / 2^{+}\left|56,{ }^{4} 10,2,2,5 / 2^{+}\right\rangle$with the chiral quark model, and the width of $\left|70,{ }^{2} 10,2,2,5 / 2^{+}\right\rangle$is predicted to be $\sim 12 \mathrm{MeV}$, which is about a factor 4 smaller than the width of $\Omega(2250)$.

The $J^{P}=3 / 2^{+}$state $\left|70,{ }^{2} 10,2,2,3 / 2^{+}\right\rangle$is a narrow state with a width of $\Gamma \simeq 23 \mathrm{MeV}$, and has comparable decay rates into $\Xi K$ and $\Xi(1530) K$ channels. These predictions of the ${ }^{3} P_{0}$ model are consistent with those in the chiral quark model [17]. While the other $J^{P}=3 / 2^{+}$ state $\left|56,{ }^{4} 10,2,2,3 / 2^{+}\right\rangle$is found to be a broad state with a width of $\Gamma \simeq 130 \mathrm{MeV}$, the partial width ratio between $\Xi K$ and $\Xi(1530) K$ is predicted to be

$$
\frac{\Gamma[\Xi K]}{\Gamma[\Xi(1530) K]} \simeq 1.4 \text {. }
$$

The predicted width for $\left|56,{ }^{4} 10,2,2,3 / 2^{+}\right\rangle$in this work is about a factor 3 larger than that predicted with the chiral quark model [17].

The $J^{P}=1 / 2^{+}$state $\left|56,{ }^{4} 10,2,2,1 / 2^{+}\right\rangle$is the broadest state in the $1 D$-wave states. It has a width of $\Gamma \sim 170 \mathrm{MeV}$, and mainly decays into the $\Xi K$ channel. However, in the chiral quark model a relatively narrower width $\Gamma \sim$ $56 \mathrm{MeV}$ is given for $\left|56,{ }^{4} 10,2,2,1 / 2^{+}\right\rangle$[17].

The $J^{P}=7 / 2^{+}$state $\left|56,{ }^{4} 10,2,2,7 / 2^{+}\right\rangle$may be a narrow state with a width of $\Gamma \sim 30 \mathrm{MeV}$. This state mainly decays into the $\Xi K$ channel. The decay properties of $\left|56,{ }^{4} 10,2,2,7 / 2^{+}\right\rangle$predicted in this work are consistent with those of chiral quark model in Ref. [17].

As a whole most of the $1 D$-wave states has a relatively narrow width, they has potentials to be observed in their dominant decay modes. The $\Omega(2250)$ resonance may be assigned to the $J^{P}=5 / 2^{+}$state $\left|56,{ }^{4} 10,2,2,5 / 2^{+}\right\rangle$or $\left|70,{ }^{2} 10,2,2,5 / 2^{+}\right\rangle$. Although the decay widths predicted for the $D$-wave states within the chiral quark model and ${ }^{3} P_{0}$ model show some differences, the partial width ratios of $\Gamma[\Xi K] / \Gamma[\Xi(1530) K]$ predicted within these two models are in a reasonable agreement with each other.

\section{SUMMARY}

Stimulated by the newly discovered $\Omega(2012)$ resonance at Belle II, in this work we have studied the OZI allowed strong decays of $1 P$ - and $1 D$-wave $\Omega$ baryons within the ${ }^{3} P_{0}$ model.

It is found that the newly observed state $\Omega(2012)$ favors the $1 P$-wave $\Omega$ state with $J^{P}=3 / 2^{-},\left|70,{ }^{2} 10,1,1,3 / 2^{-}\right\rangle$. Both the decay mode and decay width are consistent with the observations. The other $1 P$-wave $\Omega$ state with $J^{P}=1 / 2^{-},\left|70,{ }^{2} 10,1,1,1 / 2^{-}\right\rangle$, might have a relatively broad width of $\mathcal{O}(10) \mathrm{MeV}$. This $J^{P}=1 / 2^{-}$state should be observed in the $\Xi^{0} K^{-}$and $\Xi^{-} \bar{K}^{0}$ channels as well.

In the $1 D$-wave $\Omega$ states, it is found that the $\Omega(2250)$ state may favor the $J^{P}=5 / 2^{+}$state $\left|56,{ }^{4} 10,2,2,5 / 2^{+}\right\rangle$or $\left|70,{ }^{2} 10,2,2,5 / 2^{+}\right\rangle$. These two $J^{P}=5 / 2^{+}$states dominantly decay into the $\Xi(1530) K$ channel, and have a similar decay width to that of $\Omega(2250)$. Due to a large uncertainty of the width of $\Omega(2250)$, we cannot distinguish it belongs to the 70 multiplet or 56 multiplet. Future experiment information will help to clarify this issue. For the other $1 D$-wave $\Omega$ baryons, we recommend looking for the $J^{P}=$ $1 / 2^{+}$and $J^{P}=7 / 2^{+}$states in the $\Xi K$ decay channel and looking for the $J^{P}=3 / 2^{+}$states in both $\Xi K$ and $\Xi(1530) K$ decay channels in future experiments.

\section{ACKNOWLEDGMENTS}

This work is supported by the National Natural Science Foundation of China under Grants No. 11405053, No. 11775078, No. U1832173, and No. 11705056. This work is also in part supported by China Postdoctoral Science Foundation under Grant No. 2017M620492.

\section{APPENDIX A: THE HARMONIC OSCILLATOR WAVE FUNCTIONS}

For the $S$-wave omega baryon, the harmonic oscillator wave function is

$$
\begin{aligned}
\Psi(0,0,0,0)= & \left(\frac{4}{\sqrt{\pi}}\right)^{\frac{1}{2}}\left(\frac{1}{\beta_{\rho}}\right)^{\frac{3}{2}} y_{0,0}\left(\vec{p}_{\rho}\right) e^{-\frac{\vec{p}_{\rho}^{2}}{2 \beta_{\rho}^{2}} \frac{\vec{p}_{\lambda}^{2}}{2 \beta_{\lambda}^{2}}} \\
& \times\left(\frac{4}{\sqrt{\pi}}\right)^{\frac{1}{2}}\left(\frac{1}{\beta_{\lambda}}\right)^{\frac{3}{2}} y_{0,0}\left(\vec{p}_{\lambda}\right)
\end{aligned}
$$


For the $P$-wave omega baryon, the harmonic oscillator wave function is

$$
\begin{aligned}
\Psi\left(0,0,1, m_{l \lambda}\right)= & -i\left(\frac{4}{\sqrt{\pi}}\right)^{\frac{1}{2}}\left(\frac{1}{\beta_{\rho}}\right)^{\frac{3}{2}} y_{0,0}\left(\vec{p}_{\rho}\right) e^{-\frac{\vec{p}_{\rho}^{2}}{2 \beta_{\rho}^{2}} \frac{\vec{p}_{\lambda}^{2}}{2 \beta_{\lambda}^{2}}} \\
& \times\left(\frac{8}{3 \sqrt{\pi}}\right)^{\frac{1}{2}}\left(\frac{1}{\beta_{\lambda}}\right)^{\frac{5}{2}} y_{1, m_{l \lambda}}\left(\vec{p}_{\lambda}\right), \\
\Psi\left(1, m_{l \rho}, 0,0\right)= & -i\left(\frac{8}{3 \sqrt{\pi}}\right)^{\frac{1}{2}}\left(\frac{1}{\beta_{\rho}}\right)^{\frac{5}{2}} y_{1, m_{l \rho}}\left(\vec{p}_{\rho}\right) e^{-\frac{\vec{p}_{\rho}^{2}}{2 \beta_{\rho}^{2}}-\frac{\vec{p}_{\lambda}^{2}}{2 \beta_{\lambda}^{2}}} \\
& \times\left(\frac{4}{\sqrt{\pi}}\right)^{\frac{1}{2}}\left(\frac{1}{\beta_{\lambda}}\right)^{\frac{3}{2}} y_{0,0}\left(\vec{p}_{\lambda}\right),
\end{aligned}
$$

For the $D$-wave omega baryon, the harmonic oscillator wave function is

$$
\begin{aligned}
\Psi\left(0,0,2, m_{l \lambda}\right)= & -\left(\frac{4}{\sqrt{\pi}}\right)^{\frac{1}{2}}\left(\frac{1}{\beta_{\rho}}\right)^{\frac{3}{2}} y_{0,0}\left(\vec{p}_{\rho}\right) e^{-\frac{\vec{p}_{\rho}^{2}}{2 \beta_{\rho}^{2}}-\frac{\vec{p}_{\lambda}^{2}}{2 \beta_{\lambda}^{2}}} \\
& \times\left(\frac{16}{15 \sqrt{\pi}}\right)^{\frac{1}{2}}\left(\frac{1}{\beta_{\lambda}}\right)^{\frac{7}{2}} y_{2, m_{l \lambda}}\left(\vec{p}_{\lambda}\right), \\
\Psi\left(2, m_{l \rho}, 0,0\right)= & -\left(\frac{16}{15 \sqrt{\pi}}\right)^{\frac{1}{2}}\left(\frac{1}{\beta_{\rho}}\right)^{\frac{7}{2}} y_{2, m_{l \rho}}\left(\vec{p}_{\rho}\right) e^{-\frac{\vec{p}_{\rho}^{2}}{2 \beta_{\rho}^{2}} \frac{\vec{p}_{\lambda}^{2}}{2 \beta_{\lambda}^{2}}} \\
& \times\left(\frac{4}{\sqrt{\pi}}\right)^{\frac{1}{2}}\left(\frac{1}{\beta_{\lambda}}\right)^{\frac{3}{2}} y_{0,0}\left(\vec{p}_{\lambda}\right),
\end{aligned}
$$

Where $\vec{p}_{\rho}=\frac{1}{\sqrt{2}}\left(\vec{p}_{1}-\vec{p}_{2}\right)$ and $\vec{p}_{\lambda}=\sqrt{\frac{1}{6}}\left(\vec{p}_{1}+\vec{p}_{2}-2 \vec{p}_{3}\right)$ are obtained in the relative Jacobi coordinates. The $y_{l, m_{l}}(\vec{p})$ is the solid harmonic polynomial.

The wave function of the meson in our calculation is

$$
\Psi(0,0)=\left(\frac{R^{2}}{\pi}\right)^{\frac{3}{4}} e^{-\frac{\left(\vec{p}_{3}-\vec{p}_{5}\right)^{2} R^{2}}{8}}
$$

Here $\vec{p}_{C}=\frac{\vec{p}_{3}-\vec{p}_{5}}{2}, R=\frac{1}{\beta_{C}}$.

\section{APPENDIX B: THE MOMENTUM SPACE INTEGRATION}

The momentum space integration $\Pi\left(L_{\rho}^{A}, M_{\rho}^{A}, L_{\lambda}^{A}, M_{\lambda}^{A}, m\right)$ are presented in the following

$$
\Pi(0,0,0,0,0)=\beta|\vec{p}| \Delta_{0,0} .
$$

For the $P$-wave omega baryon decay,

$$
\Pi(0,0,1,0,0)=\left(\frac{1}{\sqrt{6} \lambda_{2}}-\frac{\lambda_{3}}{2 \lambda_{2}} \beta|\vec{p}|^{2}\right) \Delta_{0,1},
$$

$\Pi(0,0,1,1,-1)=-\frac{1}{\sqrt{6} \lambda_{2}} \Delta_{0,1}=\Pi(0,0,1,-1,1)$.

For the $D$-wave omega baryon decay,

$\Pi(0,0,2,0,0)=\left(\frac{\lambda_{3}^{2}}{2 \lambda_{2}^{2}} \beta|\vec{p}|^{3}-\frac{\sqrt{6} \lambda_{3}}{3 \lambda_{2}^{2}}|\vec{p}|\right) \Delta_{0,2}$,

$\Pi(0,0,2,1,-1)=\frac{\lambda_{3}}{\sqrt{2} \lambda_{2}^{2}}|\vec{p}| \Delta_{0,2}=\Pi(0,0,2,-1,1)$.

Here,

$\lambda_{1}=\frac{1}{2 \beta_{\rho}^{2}}+\frac{1}{2 \beta_{\rho}^{\prime 2}}, \quad \lambda_{2}=\frac{1}{2 \beta_{\lambda}^{2}}+\frac{1}{2 \beta_{\lambda}^{\prime 2}}+\frac{R^{2}}{3}$,

$\lambda_{3}=\frac{\sqrt{6} m_{3}}{\left(2 m_{3}+m_{5}\right) \beta_{\lambda}^{\prime 2}}+\frac{m_{3}}{m_{3}+m_{5}} \frac{\sqrt{6} R^{2}}{3}$,

$\lambda_{4}=\frac{3 m_{3}^{2}}{\left(2 m_{3}+m_{5}\right)^{2} \beta_{\lambda}^{\prime 2}}+\frac{m_{3}^{2}}{\left(m_{3}+m_{5}\right)^{2}} \frac{R^{2}}{2}$,

$\beta=1-\frac{\lambda_{3}}{\sqrt{6} \lambda_{2}}$,

and

$$
\begin{aligned}
\Delta_{0,0}= & \left(\frac{1}{\pi \beta_{\rho}^{\prime 2}}\right)^{\frac{3}{4}}\left(\frac{1}{\pi \beta_{\lambda}^{\prime 2}}\right)^{\frac{3}{4}}\left(\frac{R^{2}}{\pi}\right)^{\frac{3}{4}}\left(\frac{\pi^{2}}{\lambda_{1} \lambda_{2}}\right)^{\frac{3}{2}} e^{-\left(\lambda_{4}-\frac{\lambda_{3}^{2}}{4 \lambda_{2}}\right)|\vec{p}|^{2}} \\
& \times\left(-\sqrt{\frac{3}{4 \pi}}\right)\left(\frac{1}{\pi \beta_{\rho}^{2}}\right)^{\frac{3}{4}}\left(\frac{1}{\pi \beta_{\lambda}^{2}}\right)^{\frac{3}{4}}, \\
\Delta_{0,1}= & \left(\frac{1}{\pi \beta_{\rho}^{\prime 2}}\right)^{\frac{3}{4}}\left(\frac{1}{\pi \beta_{\lambda}^{\prime 2}}\right)^{\frac{3}{4}}\left(\frac{R^{2}}{\pi}\right)^{\frac{3}{4}}\left(\frac{\pi^{2}}{\lambda_{1} \lambda_{2}}\right)^{\frac{3}{2}} e^{-\left(\lambda_{4}-\frac{\lambda_{3}^{2}}{4 \lambda_{2}}\right)|\vec{p}|^{2}} \\
& \times \frac{3 i}{4 \pi}\left(\frac{1}{\pi \beta_{\rho}^{2}}\right)^{\frac{3}{4}}\left(\frac{8}{3 \sqrt{\pi}}\right)^{\frac{1}{2}}\left(\frac{1}{\beta_{\lambda}^{2}}\right)^{\frac{5}{4}}, \\
\Delta_{0,2}= & \left(\frac{1}{\pi \beta_{\rho}^{\prime 2}}\right)^{\frac{3}{4}}\left(\frac{1}{\pi \beta_{\lambda}^{\prime 2}}\right)^{\frac{3}{4}}\left(\frac{R^{2}}{\pi}\right)^{\frac{3}{4}}\left(\frac{\pi^{2}}{\lambda_{1} \lambda_{2}}\right)^{\frac{3}{2}} e^{-\left(\lambda_{4}-\frac{\lambda_{3}^{2}}{4 \lambda_{2}}\right)|\vec{p}|^{2}} \\
& \times \frac{\sqrt{15}}{8 \pi}\left(\frac{1}{\pi \beta_{\rho}^{2}}\right)^{\frac{3}{4}}\left(\frac{16}{15 \sqrt{\pi}}\right)^{\frac{1}{2}}\left(\frac{1}{\beta_{\lambda}^{2}}\right)^{\frac{7}{4}},
\end{aligned}
$$

where the momentum space integration $\Pi\left(L_{\rho}^{A}, M_{\rho}^{A}\right.$, $\left.L_{\lambda}^{A}, M_{\lambda}^{A}, m\right)$ is introduced in Ref. [41], what makes our differences from Ref. [41] is that we take into account difference of the masses of the quarks in baryons. 
[1] V. E. Barnes et al., Observation of a Hyperon with Strangeness -3, Phys. Rev. Lett. 12, 204 (1964).

[2] Y. Oh, Xi and omega baryons in the Skyrme model, Phys. Rev. D 75, 074002 (2007).

[3] S. Capstick and N. Isgur, Baryons in a relativized quark model with chromodynamics, Phys. Rev. D 34, 2809 (1986); AIP Conf. Proc. 132, 267 (1985).

[4] R. N. Faustov and V. O. Galkin, Strange baryon spectroscopy in the relativistic quark model, Phys. Rev. D 92, 054005 (2015).

[5] U. Löring, B. C. Metsch, and H. R. Petry, The light baryon spectrum in a relativistic quark model with instanton induced quark forces: The nonstrange baryon spectrum and ground states, Eur. Phys. J. A 10, 395 (2001).

[6] J. Liu, R. D. McKeown, and M. J. Ramsey-Musolf, Global analysis of nucleon strange form factors at low $Q^{2}$, Phys. Rev. C 76, 025202 (2007).

[7] K. T. Chao, N. Isgur, and G. Karl, Strangeness -2 and -3 baryons in a quark model with chromodynamics, Phys. Rev. D 23, 155 (1981).

[8] Y. Chen and B. Q. Ma, Light flavor baryon spectrum with higher order hyperfine interactions, Nucl. Phys. A831, 1 (2009).

[9] C. S. An, B. C. Metsch, and B. S. Zou, Mixing of the lowlying three- and five-quark $\Omega$ states with negative parity, Phys. Rev. C 87, 065207 (2013).

[10] C. S. An and B.S. Zou, Low-lying $\Omega$ states with negative parity in an extended quark model with Nambu-JonaLasinio interaction, Phys. Rev. C 89, 055209 (2014).

[11] C. Hayne and N. Isgur, Beyond the wave function at the origin: Some momentum dependent effects in the nonrelativistic quark model, Phys. Rev. D 25, 1944 (1982).

[12] M. Pervin and W. Roberts, Strangeness -2 and -3 baryons in a constituent quark model, Phys. Rev. C 77, 025202 (2008).

[13] G. P. Engel, C. B. Lang, D. Mohler, and A. Schafer, QCD with two light dynamical chirally improved quarks, Acta Phys. Pol. B Proc. Suppl. 6, 873 (2013).

[14] J. Liang, W. Sun, Y. Chen, W.-F. Qiu, M. Gong, C. Liu, Y.-B. Liu, Z.-F. Liu, J.-P. Ma, and J.-B. Zhang (CLQCD Collaboration), Spectrum and Bethe-Salpeter amplitudes of $\Omega$ baryons from lattice QCD, Chin. Phys. C 40, 041001 (2016).

[15] C. Patrignani et al. (Particle Data Group), Review of particle physics, Chin. Phys. C 40, 100001 (2016).

[16] J. Yelton et al. (Belle Collaboration), Observation of an Excited $\Omega^{-}$Baryon, Phys. Rev. Lett. 121, 052003 (2018).

[17] L. Y. Xiao and X. H. Zhong, Possible interpretation of the newly observed $\Omega(2012)$ state, Phys. Rev. D 98, 034004 (2018).

[18] T. M. Aliev, K. Azizi, Y. Sarac, and H. Sundu, Interpretation of the newly discovered $\Omega(2012)$, Phys. Rev. D 98, 014031 (2018).

[19] T. M. Aliev, K. Azizi, Y. Sarac, and H. Sundu, Nature of the $\Omega(2012)$ through its strong decays, Eur. Phys. J. C 78, 894 (2018).

[20] M. P. Valderrama, $\Omega(2012)$ as a hadronic molecule, Phys. Rev. D 98, 054009 (2018).

[21] R. Pavao and E. Oset, Coupled channels dynamics in the generation of the $\Omega(2012)$ resonance, Eur. Phys. J. C 78, 857 (2018).
[22] Y. H. Lin and B.S. Zou, Hadronic molecular assignment for the newly observed $\Omega^{*}$ state, Phys. Rev. D 98, 056013 (2018).

[23] Y. Huang, M. Z. Liu, J. X. Lu, J. J. Xie, and L. S. Geng, Strong decay modes $\bar{K} \Xi$ and $\bar{K} \Xi \pi$ of the $\Omega(2012)$ in the $\bar{K} \Xi(1530)$ and $\eta \Omega$ molecular scenario, Phys. Rev. D 98, 076012 (2018).

[24] M. V. Polyakov, H. D. Son, B. D. Sun, and A. Tandogan, $\Omega(2012)$ through the looking glass of flavour SU(3), arXiv: 1806.04427.

[25] L. Micu, Decay rates of meson resonances in a quark model, Nucl. Phys. B10, 521 (1969).

[26] R. D. Carlitz and M. Kislinger, Regge amplitude arising from $S U(6)_{W}$ vertices, Phys. Rev. D 2, 336 (1970).

[27] A. Le Yaouanc, L. Oliver, O. Pene, and J.-C. Raynal, Naive quark pair creation model and baryon decays, Phys. Rev. D 9, 1415 (1974).

[28] A. Le Yaouanc, L. Oliver, O. Pene, and J. C. Raynal, Resonant partial wave amplitudes in $\pi+n \rightarrow \pi+\pi+n$ according to the naive quark pair creation model, Phys. Rev. D 11, 1272 (1975).

[29] A. Le Yaouanc, L. Oliver, O. Pene, and J. C. Raynal, Why Is $\psi^{\prime \prime \prime}$ (4.414) SO narrow?, Phys. Lett. 72B, 57 (1977).

[30] A. Le Yaouanc, L. Oliver, O. Pene, and J.-C. Raynal, Strong decays of $\psi^{\prime \prime}(4.028)$ as a radial excitation of charmonium, Phys. Lett. 71B, 397 (1977).

[31] A. Le Yaouanc, L. Oliver, O. Pene, and J.C. Raynal, Hadron Transitions in the Quark Model (Gordon and Breach, New York, USA, 1988), p. 311.

[32] A. Le Yaouanc, L. Oliver, O. Pene, and J. C. Raynal, Naive quark pair creation model of strong interaction vertices, Phys. Rev. D 8, 2223 (1973).

[33] Z. P. Li, The threshold pion photoproduction of nucleons in the chiral quark model, Phys. Rev. D 50, 5639 (1994).

[34] Q. Zhao, Z. p. Li, and C. Bennhold, Vector meson photoproduction with an effective Lagrangian in the quark model, Phys. Rev. C 58, 2393 (1998).

[35] X. H. Zhong, Q. Zhao, J. He, and B. Saghai, Study of $\pi^{-} p \rightarrow \eta n$ at low energies in a chiral constituent quark model, Phys. Rev. C 76, 065205 (2007).

[36] L. Y. Xiao and X. H. Zhong, Strong decays of higher excited heavy-light mesons in a chiral quark model, Phys. Rev. D 90, 074029 (2014).

[37] L. C. Gui, L.S. Lu, Q.F. L, X. H. Zhong, and Q. Zhao, Strong decays of higher charmonium states into open-charm meson pairs, Phys. Rev. D 98, 016010 (2018).

[38] S. Godfrey and K. Moats, Properties of excited charm and charm-strange mesons, Phys. Rev. D 93, 034035 (2016).

[39] L. Y. Xiao, K. L. Wang, Q.f. Lü, X. H. Zhong, and S. L. Zhu, Strong and radiative decays of the doubly charmed baryons, Phys. Rev. D 96, 094005 (2017).

[40] X. H. Zhong and Q. Zhao, Charmed baryon strong decays in a chiral quark model, Phys. Rev. D 77, 074008 (2008).

[41] L. Y. Xiao, Q. F. Lü, and S. L. Zhu, Strong decays of the $1 P$ and 2D doubly charmed states, Phys. Rev. D 97, 074005 (2018). 